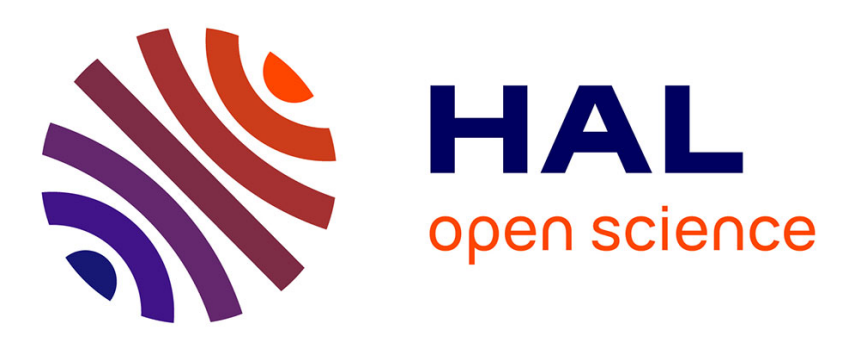

\title{
Quand le Niger aidait la France. Le Parrainage de Rosières-en-Santerre par la colonie du Niger (1942-1952) \\ Vincent Bonnecase
}

\section{To cite this version:}

Vincent Bonnecase. Quand le Niger aidait la France. Le Parrainage de Rosières-en-Santerre par la colonie du Niger (1942-1952). Afrique et histoire, 2009. halshs-01618829

\section{HAL Id: halshs-01618829 \\ https://shs.hal.science/halshs-01618829}

Submitted on 18 Oct 2017

HAL is a multi-disciplinary open access archive for the deposit and dissemination of scientific research documents, whether they are published or not. The documents may come from teaching and research institutions in France or abroad, or from public or private research centers.
L'archive ouverte pluridisciplinaire HAL, est destinée au dépôt et à la diffusion de documents scientifiques de niveau recherche, publiés ou non, émanant des établissements d'enseignement et de recherche français ou étrangers, des laboratoires publics ou privés. 


\section{QUAND LE NigER AIDAIT LA FranCE.}

\section{LE PARRAINAGE DE ROSIERES-EN-SANTERRE PAR LA COLONIE DU NIGER}

(1942-1952)

\section{VINCENT BONNECASE}

Résumé : De 1942 à 1952, la petite ville picarde de Rosières-en-Santerre, sinistrée par la guerre, reçoit une aide financière et en nature provenant de la colonie du Niger. Cette aide, financée sur fonds colonial et par des collectes privées, vise à permettre la reconstruction de la ville française et l'allocation de secours à ses pauvres. Tout en s'inscrivant dans un rapport colonial classique, cet épisode de micro-bistoire est révélateur des représentations officielles de l'aide publique et caritative qui peuvent se construire entre une métropole européenne et une colonie africaine dans les années 1940.

Abstract : From 1942 till 1952, the French town of Rosières-en-Santerre received a financial and food aid from the colony of Niger for being destroyed during the war. This aid, which was both public and private, aimed at supporting the reconstruction of the town and at supporting its poors. While joining a classic colonial relationship, this episode of micro-bistory is indicative of official representations of aid-link that could exist between a European country and its African colony in the 1940".

Après la Seconde guerre mondiale, se développe un nouveau type de rapport entre les pays industrialisés et les pays colonisés ou décolonisés, rapport que l'on désigne globalement sous la notion d'aide internationale. Jusqu'alors, l'essentiel des réalisations coloniales, en terme d'infrastructures ou d'équipements sociaux, est largement autofinancé par le biais de la fiscalité, tout au moins pour ce qui est de l'AOF'. À partir des années 1940, ces investissements proviennent essentiellement des financements métropolitains. L'idée d'une solidarité des pays riches vis-à-vis des territoires les plus démunis commence alors à se mettre en place. Cette idée, conceptualisée en terme d'aide aux pays sous-développés à la fin de la décennie ${ }^{2}$, se construit progressivement au cours de la seconde partie du XXe siècle, avec ses outils institutionnels, ses codes et ses rôles qui se fixent peu à peu entre ce qui devient le Nord et le Sud.

Rosières-en-Santerre, seule des petites villes françaises à avoir une rue du $\mathrm{Niger}^{3}$, semble porter les traces d'une solidarité de ce type entre la France et un territoire africain, mais pas dans le sens attendu. Déjà fortement éprouvée pendant la Première guerre mondiale, cette petite commune picarde de 2000 habitants est

\footnotetext{
${ }^{1}$ La loi des finances de 1900 prévoit l'autonomie financière des colonies ; C.-R. AGERON (1991 : 116).

${ }^{2}$ Le point IV du discours à la nation prononcé par le président Truman en 1948 est souvent cité comme premier texte à diffusion internationale employant le concept de pays sous-développé ; G. RIST (1996 : 116-125).

${ }^{3}$ Rosières-en Santerre, commune située à une trentaine de kilomètres à l'est d'Amiens, compte 2014 habitants avant la guerre d'après le recensement de 1936. La rue du Niger est le principal axe qui mène à l'Hôtel de ville.
} 
partiellement détruite en 1940 au moment de la bataille de la Somme. En 1941, le gouvernement de Vichy l'érige en ville martyre et met en place un système de parrainage entre collectivités pour financer sa reconstruction. En 1942, la colonie du Niger est désignée comme marraine. Le lien de parrainage, interrompu à l'issue de la même année après le ralliement de l'AOF à la France libre, est repris en 1945 et perdure jusque 1952. Pendant dix ans, Rosières-en-Santerre reçoit des fonds publics et privés en provenance du Niger. Une subvention annuelle est prélevée sur le budget territorial pour financer la reconstruction de la petite ville picarde. Parallèlement, un comité de soutien se constitue sur le territoire nigérien pour y collecter des dons auprès de particuliers. Ces dons, financiers ou en nature, sont destinés à secourir les nécessiteux de Rosièresen-Santerre.

Ce parrainage d'une commune de la France métropolitaine par un territoire africain, le moins pourvu de l'AOF ${ }^{4}$, peut paraitre paradoxal. Il découle pourtant de pratiques en usage dans les colonies. Les subventions publiques envoyées à Rosières-en-Santerre constituent une forme de contribution de la colonie aux dépenses métropolitaines. Pareil transfert financier d'une colonie à la métropole, habituellement limité, augmente pendant les deux guerres ${ }^{5}$ : dans la logique coloniale, il est légitime de faire participer les populations colonisées à l'effort militaire. Le parrainage de Rosières-en-Santerre par la colonie du Niger apparait dans cette optique comme un surcroît de dépense imposé au budget territorial nigérien en temps de guerre puis de reconstruction. Quant aux dons privés envoyés depuis le Niger à Rosières-en-Santerre, ils proviennent en grande part des Français vivant aux colonies. Celles-ci sont le terrain privilégié d'un petit nombre d'œuvres de bienfaisances qui collectent des fonds auprès de l'élite, principalement française, au bénéfice de métropolitains déshérités. Cette pratique est particulièrement activée sous le régime de Vichy qui place la charité parmi les valeurs à privilégier et tend à institutionnaliser les activités caritatives ${ }^{6}$.

La lecture officielle de ce parrainage tend cependant à en faire autre chose que la simple déclinaison de pratiques coloniales usuelles. Que ce soit du point de vue du gouvernement du Niger ou de celui de la municipalité de Rosières-en-Santerre, le parrainage est placé sous l'égide d'une solidarité nigérienne inédite à l'égard de la métropole. Les subventions publiques sont présentées comme le don de la population du Niger dans son ensemble et, les collectes privées, comme l'aide apportée par les Nigériens aux pauvres de Rosièresen-Santerre. Quand bien même l'origine nigérienne de cette aide n'existerait que dans le discours officiel, son existence, même sur le plan discursif, est significative : l'idée de solidarité d'un continent à un autre est alors assez balbutiante pour que des autorités coloniales puissent s'en réclamer en transférant des fonds d'une colonie africaine à une commune métropolitaine. Il est alors possible qu'une colonie africaine subventionne la reconstruction d'une petite ville française au nom d'une aide entre collectivités. Il est alors possible que des associations privées sollicitent, sur un territoire sahélien où une partie importante de la population est affectée

\footnotetext{
${ }^{4}$ Le Niger est notamment la colonie de l'AOF dont le budget rapporté à la population est le moins élevé.

${ }^{5}$ Les contributions financières de l'AOF aux dépenses métropolitaines, rares et faibles dans l'ensemble, représentent ainsi plus de 3\% du budget fédéral en 1915 et plus de 1\% en 1939 ; C. COQUERY-Vidrovitch $(1982$ : 239).
} 
par la malnutrition et la sous-alimentation ${ }^{7}$, des dons destinés à des métropolitains. Le parrainage de Rosièresen-Santerre par la colonie du Niger, aussi isolé soit-il, est significatif du contexte dans lequel se construit la figure émergente de l'aide dans les années 1940 : les rôles impartis par cette relation d'aide sont encore assez mal établis pour ne pas se réduire à un schéma dualiste où les pourvoyeurs de fonds seraient nécessairement au Nord et les territoires à pourvoir nécessairement au Sud.

\section{LES SUBVENTIONS NIGERIENNES A LA METROPOLE}

Les subventions publiques envoyées par la colonie du Niger à la commune de Rosières-en-Santerre relèvent d'une logique coloniale assez classique, celle du prélèvement financier opéré par une métropole sur une colonie. Ce prélèvement s'accompagne cependant d'un discours officiel de type caritatif assez nouveau qui, tant du côté des autorités coloniales que de la municipalité picarde, invite à lire les subventions, prélevées sur le budget territorial du Niger, comme l'aide d'une population africaine épargnée par la guerre à une commune française sinistrée. On peut inscrire cette représentation officielle du parrainage dans ses deux contextes de mise en œuvre, le contexte vichyssois d'une part et celui de l'après-guerre d'autre part.

\section{Une instauration vichyssoise}

La part du régime de Vichy dans le parrainage de Rosières par la colonie du Niger est extrêmement limitée en terme de transfert financier, puisqu'une seule subvention, réduite, est versée en 1942. Cette part est en revanche importante dans les modalités mêmes d'instauration du système de parrainage.

Le système de parrainage repose sur une logique de péréquation mise en place en 1941 par le gouvernement de Vichy afin d'augmenter les ressources des communes les plus touchées par la guerre. Après la bataille de la Somme en mai 1940, Rosières est détruite aux deux tiers. Elle apparaît au sous-préfet de Montdidier comme «la ville du département la plus touchée au prorata de son nombre d'habitations ${ }^{8}$ ». C'est pourquoi le préfet de la Somme saisit en 1941 le ministère de l'Intérieur afin de solliciter l'adoption de la ville picarde par une «cité n'ayant pas souffert de la guerre ${ }^{9}$ ». Les territoires de l'AOF, restés sous le contrôle de Vichy, sont alors loin des combats et, à ce titre, mis à contribution. Le fait de ponctionner plus lourdement les colonies en temps de guerre n'est pas exceptionnel, la métropole ayant recouru à pareilles ponctions en 1939 comme en $1914^{10}$. En octobre 1942, le gouverneur du Niger, Jean-François Toby, est avisé officiellement par le gouverneur général de l'AOF, Pierre Boisson, que «la colonie du Niger a été choisie comme marraine de

\footnotetext{
${ }^{6}$ M. Cointet-Labrousse $(1987: 152)$.

${ }^{7}$ Archives nationales du Burkina Faso (ANBF), 9V326, Comité inter-États de lutte contre la sécheresse dans le Sahel, Rapport sur la situation nutritionnelle dans le Sahel (1973).

8 Archives municipales de Rosières-en-Santerre (AMR), Lettre du sous-préfet de Montdidier au préfet de la Somme (06/03/1943). Sur 600 bâtiments, 248 sont complètement détruits et les deux tiers restants sont endommagés.

9 AMR, Lettre du préfet de la Somme au maire de Rosières (07/07/1942).

10 M. MiChel (2003 : 49-63) ; C. COQUERY-VidrovitCH (1992 : 244-246).
} 
Rosières », promue «ville martyre» par décret du ministère de l'Intérieur ${ }^{11}$. Une première subvention de 50000 francs CFA est versée par la colonie le même mois, suivie d'un crédit de 200000 francs CFA inscrit au budget de $1943^{12}$. Ce crédit ne peut cependant être versé du fait de l'occupation de l'Afrique du Nord par les forces alliées et le ralliement de l'AOF à la France libre en novembre 1942. L'opération est suspendue et d'autres collectivités, cette fois des communes métropolitaines ${ }^{13}$, sont désignées pour parrainer la commune picarde. Le parrainage de Rosières par la colonie du Niger sous le régime de Vichy se réduit donc à un mois de correspondance et une seule subvention de 50000 FCFA.

Aussi limité ce bilan matériel soit-il, l'instauration de ce système de parrainage est marquée par l'idéologie du régime de Vichy. Elle en porte d'abord la dimension fortement centralisatrice, dimension marquée en métropole, mais aussi «dans un contexte colonial où le principe d'autorité s'applique sans fard ${ }^{14}$ ». Le rôle du préfet de la Somme et du sous-préfet de Montdidier dans le choix de Rosières, ville sinistrée parmi d'autres, n'est pas fortuit : une nouvelle loi instituée en octobre 1940 renforce le pouvoir préfectoral au détriment des autorités municipales et départementales élues ${ }^{15}$. Quant à la désignation du Niger, elle est directement décrétée par le ministère de l'Intérieur ${ }^{16}$. Le système de parrainage prévoit, en métropole, la validation du parrainage par un vote favorable des conseils municipaux des communes désignées pour être marraines ${ }^{17}$. Mais au Niger, il n'existe nulle assemblée représentative pour ratifier la décision : le décret ministériel est simplement entériné par un gouvernement colonial resté aux ordres du gouvernement du maréchal Pétain. Et une fois le lien de parrainage institué, les modalités d'utilisation des subventions échappent totalement à la colonie donatrice. Un arrêté gouvernemental pris à Vichy en 1941 précise que « chaque fois qu'une commune adoptée obtient une aide de la cité marraine, il convient d'en informer le délégué départemental du Secours national et de procéder $[\ldots]$ à la répartition des dons reçus en accord avec lui1 ${ }^{18}$ ». Le Secours national, institution chargée d'allouer des secours, est lui-même directement contrôlé par le chef de l'État de Vichy ${ }^{19}$. L'ensemble du processus de parrainage, depuis le prélèvement de fonds jusqu'à leur redistribution, reste donc solidement encadré par l'État.

L'unanimité populaire mise en exergue par les autorités coloniales dans le cadre du parrainage est un autre élément important de l’idéologie du régime de Vichy. Dans la première lettre qu'il envoie au maire de Rosières, Gaston Villain, le gouverneur du Niger, Jean-François Toby, s'exprime «au nom de tous les Nigériens»,

11 AMR, Lettre du gouverneur du Niger au maire de Rosières (13/10/1942).

12 AMR, Lettre du sous-préfet de Montdidier au préfet de la Somme (06/03/1943).

13 AMR, Lettre du préfet de la Somme au maire de Rosières (01/05/1943). Il s'agit successivement des communes de Grazac, Aurecsur-Loire, Homecourt et Yssingeaux.

14 J. CANTIER et E. JENNINGS (2004: 8).

${ }^{15}$ La loi du 12/10/1940 suspend les sessions des conseils généraux et transfère leur pouvoir aux préfets qui contrôlent également la composition des conseils municipaux des communes de plus de 2000 habitants ; M. COINTET-LABROusSE (1987 : 48). Le maire de Rosières, Gaston Villain est ainsi nommé à ce poste par arrêté du 03/03/1941.

16 AMR, Lettre du préfet de la Somme au maire de Rosières (07/07/1942).

17 AMR, Lettre du préfet de la Somme au maire de Rosières (08/07/1943).

18 AMR, Lettre du préfet de la Somme au maire de Rosières (15/06/1943). 
stipulant qu'ils « auront à cœur de verser leur obole pour aider à la reconstruction de [la] cité si éprouvée par la guerre $^{20}$ ». Cette intégration de «tous les Nigériens » dans le parrainage est factice puisque la population n'est concernée que fiscalement, par le biais de l'impôt direct qui alimente le budget de la colonie d'où sont prélevées les subventions. Elle relève cependant d'un «sentiment de reconstruction nécessaire de la nation » fortement exacerbé par le régime de Vichy d'après lequel «les divisions antérieures sont $[\ldots]$ absolument inséparables des causes de l'effondrement ${ }^{21} »$. Le parrainage témoigne ainsi, dans cette optique officielle, d'un désir d'unité de la population nigérienne derrière le maréchal Pétain. Le gouverneur le présente comme signe " de la solidarité qui unit l'Empire et la métropole », comme signe selon lequel, «malgré la menace anglodissidente, tous les Nigériens [...] affirment leur volonté inébranlable de rester fidèles à la France du Maréchal22 ». Il importe d'autant plus de mettre en exergue la fidélité des populations nigériennes au régime de Vichy que les colonies voisines de l'AEF ont fait défection en ralliant dès août 1940 la France libre. Le gouverneur suppose en cela la prolongation dans la colonie de «l'engrenage du consentement» suscité en métropole, engrenage qui repose sur l’idée qu'il «n'existe aucune issue en dehors de celle de Vichy²3 ».

L'empreinte vichyssoise du parrainage de Rosières par la colonie du Niger tient enfin à son caractère caritatif. Le parrainage représente plus qu'une obligation budgétaire pour le gouverneur, il constitue une «magnifique œuvre d'entraide» à laquelle il affirme «sa joie et sa fierté de participer» ${ }^{24}$. Or, les activités caritatives sont hautement recommandées par le régime de Vichy pour atténuer les souffrances de la guerre, en métropole mais aussi dans les colonies. Celles-ci sont concernées par un ensemble d'arrêtés ministériels visant à favoriser et encadrer les élans caritatifs. En décembre 1940, le plan de secours national, visant à venir en aide pendant l'hiver aux populations métropolitaines éprouvées par la guerre, prévoit la collecte de fonds privés dans l'Empire ${ }^{25}$. En octobre 1941, une Commission coloniale du Secours national est chargée de coordonner l'action des organisations caritatives des colonies et de porter assistance aux coloniaux vivant en métropole, en particulier les soldats démobilisés ${ }^{26}$. En janvier 1942, il est décidé de mettre systématiquement à charge des budgets coloniaux une partie des frais de voyage des missionnaires qui, outre leur activité religieuse, assurent

${ }^{19}$ Le Secours national, créé en 1914 et suspendu à la fin de la Première guerre, est réactivé en 1939 et placé sous l'autorité du Maréchal Pétain en 1940.

20 AMR, Lettre du gouverneur du Niger au maire de Rosières (13/10/1942).

21 P. LABORIE (2001: 240).

22 AMR, Lettre du gouverneur du Niger au maire de Rosières (13/10/1942). Jean-François Toby n'apparaît pourtant pas parmi les administrateurs les plus soumis au régime de Vichy. Après le ralliement de l'AOF à la France libre, il demande d'abord d'être relevé de son poste avant d'y être finalement maintenu, contrairement au gouverneur général Pierre Boisson, évincé en juillet 1943 ; C. AKPO-VACHE (1996:174).

23 P. LABORIE (2001: 239).

24 AMR, Lettre du gouverneur du Niger au maire de Rosières (13/10/1942).

25 Centre des archives d'outre-mer (CAOM), 1affpol/870, Secrétariat aux colonies, Direction des affaires politiques, Plan de secours national aux populations éprouvées par la guerre pour l'hiver 1940-1941 (décembre 1940). Les destinataires privilégiés de ces secours sont les réfugiés, les sinistrés, les démobilisés, les familles de prisonniers de guerre, les veuves et les orphelins de guerre, les familles nombreuses, les malades et les infirmes.

26 CAOM, 1affpol/870, Commission coloniale du Secours national, Rapport sur l'activité de la Commission depuis sa création (31/12/1942). Environ 3500 coloniaux sont secourus par le Secours national d'octobre 1941 à décembre 1942. 
une fonction médicale et éducative ${ }^{27}$. En échange de ce soutien de l'État, ces missionnaires se doivent «d'assurer [la] révolution nationale dans l'Empire ${ }^{28}$ ». Le parrainage de Rosières par la colonie du Niger s'inscrit dans cette série de mesures prises par le gouvernement de Vichy pour développer et institutionnaliser les pratiques caritatives dans les colonies comme dans la métropole.

Ainsi, même si le parrainage de Rosières par la colonie du Niger n'a, sous le régime de Vichy, quasiment aucune implication matérielle, il reste significatif de la volonté de ce régime d'exporter certains éléments de la révolution nationale dans les colonies.

\section{Les subventions nigériennes d'après-guerre}

En 1945, le gouvernement du Niger restaure le lien de parrainage avec la commune de Rosières, lien qui perdure jusque 1952. Cela donne lieu au versement régulier de subventions grâce auxquelles Rosières est reconstruite. Cette reprise du parrainage se fait dans un contexte d'émergence de l'aide internationale, de ses institutions et de ses flux financiers. Et tout en s'inscrivant dans une logique coloniale de prélèvement de ressources au bénéfice de la métropole, le parrainage est officiellement présenté comme relevant de l'aide par les autorités qui le remettent en œuvre.

Les modalités de mise en œuvre du parrainage en 1945 ne s'inscrivent que partiellement dans celles qui prévalent trois ans avant, même si un des protagonistes de cette mise en œuvre est resté le même, le gouverneur Jean-François Toby. En 1942, l'institution du parrainage est impulsée par le gouvernement métropolitain. En 1945, sa restauration est suscitée par le gouvernement colonial sans que cela réponde à une demande du ministère de la France d'Outre-mer. Le gouverneur général de l'AOF, Pierre Cournarie, invite alors non seulement le Niger, mais aussi d'autres colonies de la Fédération, à soutenir des villes françaises sinistrées par la guerre ${ }^{29}$. En mars 1945, le gouverneur du Niger, Jean-François Toby, prend la décision de lancer une "campagne de secours en faveur de [...] Rosières-en-en Santerre, filleule [...] du Niger», en se référant à l'adoption de la commune à laquelle il avait lui-même contribué en 194230. Le versement de subventions annuelles, d'abord arrêté par le gouvernement du Niger en 1945, est ensuite validé par le Conseil général créé après la guerre dans le cadre des nouvelles institutions de l'Union française. De 1948 à 1952, il fait ainsi l'objet d'un vote de cette assemblée à l'issu de délibérations budgétaires. Certes, le Conseil général est très faiblement représentatif, une minorité de la population participant aux élections de janvier 1947 organisées

27 CAOM, 1affpol/476, Secrétaire d'État aux colonies, Direction des affaires politiques, Rapport sur les subventions aux œuvres sociales privées des colonies (15/12/1942). Cet arrêté du 19/01/1942 inscrit au chapitre 60 des budgets coloniaux le remboursement du quart des frais de voyage des missionnaires, un autre quart devant être financé par les compagnies maritimes.

28 CAOM, 1affpol/2286, Secrétariat aux colonies, Direction des affaires politiques, Rapport sur l'activité de la congrégation des sœurs missionnaires de Notre-Dame des apôtres en AOF de 1939 à 1942 (05/10/1942).

29 AMR, Lettre du ministre de la FOM au gouverneur général de l'AOF (14/03/1947). Cette lettre mentionne le Dahomey et le Soudan comme autres colonies marraines sans préciser les villes destinataires de l'aide.

30 Archives nationales du Niger (ANN), 2H1.7, Gouvernement du Niger, Arrêté du 21/03/1945. 
selon un principe de double collège et largement ignorées par les ruraux ${ }^{31}$. Le rôle d'une assemblée locale dans le parrainage n'en donne pas moins à ce dernier une légitimité populaire, aussi minime soit-elle, et laisse accroire que l'aide fournie est d'origine nigérienne. De 1945 à 1952, le nouveau maire de Rosières, André Masson, ne manque jamais, dans chacune de ses lettres, d'adresser ses remerciements à «la population du Niger» qui «comprend l'infortune d'une ville détruite aux trois quarts» et «ne cesse de donner une aide généreuse dans des moments difficiles $»^{32}$.

En terme de volume financier, les subventions d'après-guerre, sont autrement plus importantes que celle versée en 1942. Un premier crédit de 600000 francs CFA est accordé en 1945. Il correspond au cumul des subventions de 200000 francs CFA pour les années 1943, 1944 et 1945, montant annuel fixé sous le gouvernement de Vichy sans pouvoir être acquitté33. Cette subvention de 200000 francs CFA est renouvelée en 1946, portée à 400000 francs CFA en 1947 et à 500000 francs CFA en 1948, soit un million de francs métropolitains, montant accordé à nouveau en 194934. À partir de là, la subvention annuelle diminue, n'atteignant plus que 100000 francs CFA en 1950 et 50000 francs CFA en 1951 et 1952, dernière année de versement. En termes relatifs, ces versements annuels constituent à l'échelle de Rosières une somme importante puisqu'elle correspond en 1948, année où l'apport est le plus élevé, au quart des recettes du budget communal ${ }^{35}$. Le maire André Masson convient lui-même disposer, grâce à l'aide du Niger, de possibilités financières sur lesquelles ne peuvent compter les communes avoisinantes ${ }^{36}$. À l'échelle du Niger en revanche, la subvention reste limitée, représentant la même année moins de $0,15 \%$ du budget territorial ${ }^{37}$.

Quoique relativement limitées, ces subventions nigériennes à la métropole sont remarquables en ce qu'elles vont à contre-sens des flux financiers qui se mettent tout juste en place après la guerre, flux qui se fixent dans un sens qui va de la métropole aux colonies. En 1945, les contributions exceptionnelles de guerre, qui en AOF avaient suscité une capitation supplémentaire, sont abrogées, les budgets territoriaux étant désormais supposés se cantonner à des dépenses opérées sur le territoire colonial concerné38. Les subventions annuelles envoyées par le Niger à la commune de Rosières constituent en cela un rare cas de prélèvement financier effectué sur une colonie au bénéfice direct d'une collectivité métropolitaine après la guerre. Dans le sens inverse, les territoires de l'AOF se mettent à bénéficier d’un appui financier de la métropole dans le cadre de la Caisse centrale de la France d'outre-mer et du Fonds d'investissement pour le Développement économique et social,

31 C. Coquery-Vidrovitch (1992: 248-250). Ce n'est qu'avec la loi cadre de 1956 que les assemblées territoriales sont élues au suffrage universel par un collège unique.

32 AMR, Lettres du maire de Rosières au gouverneur du Niger (27/12/1945 et 19/11/1946).

33 AMR, Lettre du gouverneur du Niger au maire de Rosières (21/03/1945).

34 AMR, Lettre du gouverneur du Niger au maire de Rosières (1945-1952). 1 franc CFA vaut 1,70 francs métropolitains jusqu’octobre 1948 et 2 francs métropolitains après.

35 AMR, Budget communal, recettes du budget primitif (1948).

36 AMR, Lettre du maire de Rosières au gouverneur du Niger (31/03/1949).

37 CAOM, 1 affpol/947, Budgets locaux (1948). Ce budget est de 359 millions de francs CFA.

${ }^{38}$ Une capitation de 10 francs a été prélevée en 1943 et 1944 en AOF pour soutenir l'effort de guerre ; C.-R. AGERON, C. COQUERYVidrovitch, G. Meynier et J. Thobie (1990 : 344). Après la guerre, les seuls fonds prélevés sur un territoire de l'AOF pouvant être dépensés sur un autre territoire sont les recettes de la fiscalité indirecte qui alimentent le budget de la Fédération. 
respectivement créés en 1944 et 1946. Ces institutions prennent en charge l'essentiel des dépenses d'équipement au Niger à partir de 1947, année de lancement du premier plan de développement en $\mathrm{AOF}^{39}$. Cette nouvelle politique française d'investissement public en Afrique s'accompagne d'un discours nouveau du ministère de la France d'Outre-mer relatif à l'aide à apporter aux pays démunis, discours s'inscrivant dans l’idéologie du développement et de l'aide internationale qui se constitue après la guerre ${ }^{40}$.

Or, au sortir de la guerre, ce nouveau paradigme de l'aide internationale ne fait qu'émerger. Même si les nouvelles institutions donnent lieu à une rupture brutale des relations financières entre la métropole et ses colonies africaines, celles-ci et celle-là ne sont pas d'emblée figées, au niveau des représentations collectives, dans les positions respectives du riche bailleur de fonds et des territoires déshérités à secourir. La proximité de la guerre autorise les autorités qui restaurent le parrainage de Rosières par le Niger à suggérer un rapport inverse. En 1946, le gouverneur général de l'AOF, Pierre Cournarie, peut ainsi expliquer le parrainage, dans une lettre envoyée au ministère de la France d'Outre-mer, par «les charges accrues [que] doivent supporter $[\ldots]$ les populations sinistrées $[\ldots]$ du fait des meurtrissures de la guerre dont les effets n'ont été que lointains en $\mathrm{AOF}^{41}$ » La même année, la visite du gouverneur du Niger, Jean-François Toby, en Picardie donne lieu à des festivités célébrant l'aide apportée par le Niger à Rosières. À cette occasion, une statue en marbre est offerte par la commune au gouverneur. Cette œuvre est celle d'un marbrier picard sélectionné après l'examen de plusieurs propositions. Elle représente, d'après les termes de son concepteur, «une femme africaine» contemplant «avec tendresse » trois roses qu'elle tient dans la main ${ }^{42}$. Elle est reproduite sur une carte postale largement diffusée. D’après la légende, la femme personnifie «le riche et compatissant Niger» et, les trois roses, Rosières refleurissant, «rose d'hier meurtrie, [...] rose d'aujourd'hui revivifiée par l'aide du Niger [et] rose de demain ressuscitée par l'aide fraternelle du Niger ». L'œuvre est présentée par son concepteur comme «allégorie sur l'entraide internationale ${ }^{43}$ ». Cette allégorie, qui date de l'année de la création du FIDES, établit bien un rapport inverse à celui qui commence alors à se constituer en terme d'aide : la colonie africaine est placée dans la position de l'aidant et le territoire métropolitain dans celui de l'aidé.

Ainsi, le parrainage de Rosières par la colonie du Niger, dans sa face publique, relève de pratiques coloniales classiques tout en étant placé sous l'égide du paradigme émergent de l'aide. Il est une forme de prélèvement financier opéré sur une colonie par la métropole au nom de la solidarité qui unit l'Empire, solidarité particulièrement invoquée par le gouvernement de Vichy instituant le parrainage. Et il est à la fois officiellement désigné comme une aide apportée par un territoire africain à une métropole sinistrée, à un moment où le rapport entre la France et ses colonies commencent à peine à se conceptualiser en terme d'aide

\footnotetext{
39 Cet appui, quoique plus limité et plus tardif au Niger que dans les colonies voisines, représente près de 800 millions de francs métropolitains de 1948 à 1958 ; S. DuluCQ $(1997$ : 234).

40 G. RIST (1996).

${ }^{41}$ ANN, 2H1.7, Télégramme du gouverneur général au ministre des colonies (14/03/1946).

42 AMR, Proposition de Gérard Ansart, architecte décorateur à Amiens, pour le cadeau de la ville de Rosières au Niger (13/06/1946).

43 AMR, Carte postale éditée en souvenir de la visite à Rosières du gouverneur du Niger (07/07/1946). La carte figure à la fin de ce texte.
} 
et de développement. Ce concept d'aide apparait alors suffisamment nouveau pour être utilisé dans un sens et dans l'autre.

\section{CHARITE ET AIDE PRIVEE AUX COLONIES}

Le parrainage de Rosières-en-Santerre par la colonie du Niger ne se réduit pas à un versement de subventions publiques. Il induit également, à partir de 1945, une aide privée coordonnée par un comité de soutien à Rosières. Celui-ci est chargé de récolter dans la colonie des fonds et des produits destinés à être redistribués à des nécessiteux de la commune picarde. Là encore, cette aide apportée d’Afrique à la métropole, tout aussi insolite qu'elle peut rétrospectivement paraittre, relève de pratiques usitées dans les colonies. Avant la guerre, les élites, principalement françaises, y sont occasionnellement sollicitées par quelques œuvres de bienfaisance qui portent secours à des métropolitains déshérités. Dans l'immédiat après-guerre, ces pratiques persistent : il est possible de collecter des fonds dans un territoire africain pour venir en aide à des populations de la France métropolitaine. L'activité caritative dans les colonies n'est pas prioritairement orientée vers les populations locales.

\section{Donataires français et donateurs du Niger}

L'aide privée récoltée à partir de 1945 au Niger au bénéfice de Rosières constitue une part importante de l'aide totale versée depuis la colonie à la commune picarde. Même si elle est essentiellement le fait des Français du Niger, elle est également sollicitée auprès de populations nigériennes et ne relève pas, dans l'optique de ses initiateurs, du seul principe de solidarité nationale.

En mars 1945, le gouverneur du Niger, Jean-François Toby, met en place un comité chargé d'organiser une «campagne de secours pour venir en aide à [la] commune en dehors des inscriptions budgétaires ${ }^{44}$ » Le comité de soutien à Rosières assure entre 1945 et 1952 deux types de dons, en nature et en espèces. En espèces, les dons annuels oscillent entre 50000 et 200000 francs CFA. Ils proviennent majoritairement de la capitale mais également du reste du territoire. Ils sont destinés par le comité soit à compléter les fonds publics dans la perspective des réparations, soit et plus largement à être "distribués parmi les nécessiteux de la ville $^{45}$ ». Quant aux dons en nature, il s'agit essentiellement de denrées alimentaires produites au Niger ou dans les autres colonies de la Fédération. Le principal de ces dons est versé en avril 1947 : une tonne de riz, 600 litres d'huile et 500 kilogrammes de café sont acheminés de Niamey à Rosières. L'accès à ces aliments est alors difficile en France, toujours soumise au rationnement. La municipalité indique ainsi en annonçant la distribution que la population de Rosières est «totalement privée de riz depuis sept ans ${ }^{46}$ ». L'organisation de cette distribution donne de fait l'image d'une population vivant dans la pénurie : l'ensemble des denrées est

44 AMR, Lettre du gouverneur du Niger au maire de Rosières (21/03/1945).

45 AMR, Lettre du gouverneur du Niger au maire de Rosières (27/09/1946).

46 AMR, Municipalité de Rosières, Avis à la population (avril 1947). 
amené sur la place publique de la commune où les habitants sont appelés à se rendre par la direction du Ravitaillement de la Somme ${ }^{47}$. Les habitants, convoqués sur plusieurs jours selon leur initiale pour éviter les trop longues files d'attente, sont priés de se munir de leur récipient d'huile et de leurs cartes d'alimentation ${ }^{48}$.

Si ces dons proviennent bien du Niger, leur financement n'est que très partiellement le fait de la population nigérienne. La plupart des fonds sont collectés dans des lieux de sociabilité réservés à l'élite de la colonie, et en particulier à l'élite expatriée. Le comité de soutien à Rosières cite ainsi, parmi les principales sources de financement, des réunions mondaines telles que les «manifestations hippiques et artistiques » ou les « soirées de gala $»^{49}$, soit autant de lieux fréquentés par la communauté française. Les membres du comité de soutien à Rosières sont de fait majoritairement français et le président en est le gouverneur. Cette collecte de dons par une organisation caritative européenne auprès de la communauté expatriée et à l'intention d'une population métropolitaine n'est pas nouvelle. Les quelques organisations présentes en Afrique avant la guerre, telles que la Croix Rouge ou Save the Children, voient d'abord le continent comme «source possible de revenus à destination des activités menées en Europe ${ }^{50} »$. L'une des activités du comité dakarois de la Croix Rouge consiste ainsi à collecter des vêtements ou des vivres pour les redistribuer à des pauvres, «européens ou assimilés ${ }^{51}$ ». Cette pratique persiste dans l'immédiat après-guerre, notamment dans les organisations de soutien aux victimes de guerre ou aux victimes de catastrophes. En 1947, l'explosion d'un navire dans le port de Brest donne ainsi lieu à une collecte de fonds organisée par l'Amicale des Bretons de Dakar dans toute l'AOF, près de 150000 FCFA étant récoltés à cet effet au Niger ${ }^{52}$.

L'aide privée sollicitée après la guerre au bénéfice de métropolitains ne se réduit cependant pas à cette dimension communautaire, le cercle des donateurs excédant celui des ressortissants français. Une élite sociale nigérienne est intégrée dans les réseaux de sociabilité fréquentés par les expatriés et participe en cela à la collecte de fonds en faveur de Rosières. Certaines collectes sont même exclusivement le fait de cette élite sociale nigérienne. En 1947, les représentants nigériens siégeant au Conseil général de la colonie décident ainsi de renoncer à leurs indemnités de session au bénéfice des « nécessiteux de la ville martyre de Rosières, filleule du Niger ${ }^{53}$ ». L'année suivante, à l'occasion du centenaire de l'abolition de l'esclavage, le comité de soutien demande à tous les chefs de circonscription de faire procéder à une souscription en faveur des œuvres d'assistance de Rosières. Les fonds sont envoyés de l'ensemble du territoire, excepté des neuf postes les plus reculés. Or la plupart des cercles, hormis ceux de la capitale et des trois principales villes du territoire, ne

\footnotetext{
47 AMR, Lettre de la Direction du ravitaillement général de la Somme au maire de Rosières (10/04/1947).

48 AMR, Municipalité de Rosières, Avis à la population (avril 1947).

49 AMR, Lettre du gouverneur du Niger au maire de Rosières (06/04/1946).

50 J. ILIFFE (1987 : 199).

51 Archives nationales du Mali (ANM), 2H2, Rapport d'activités de la Croix Rouge de Dakar (22/04/1936).

52 ANN, 2H1.7, Lettre du président de l'Amicale des bretons de Dakar au gouverneur du Niger (06/08/1947).

53 AMR, Lettre du président du Conseil général du Niger au maire de Rosières (11/04/1947).
} 
comptent pas de ressortissants français, sinon les administrateurs coloniaux ${ }^{54}$. À la même occasion, des collectes sont organisées dans les écoles nigériennes. Sur un territoire qui compte un taux de scolarisation de moins de $2 \% 55$, les familles visées par ces collectes appartiennent là encore aux catégories les plus fortunées de la population mais il s'agit majoritairement, d'après l'onomastique des donateurs, de familles nigériennes ${ }^{56}$.

Même si cela reste plus marginal, les collectes de dons à l'intention de métropolitains peuvent aussi viser une population nigérienne plus large que la seule élite habilitée à fréquenter les cercles expatriés. Il arrive notamment que l'on sollicite des dons sur la voie publique, pratique usitée par les différents comités locaux de la Croix Rouge, pendant la guerre ${ }^{57}$. On ne trouve pas trace de cette pratique dans le cadre du parrainage de Rosières mais dans un autre cas similaire. En 1954, un comité semblable à celui de Rosières se met ainsi en place au Niger, suite à un séisme à Orléansville, ville algérienne peuplée par de nombreux ressortissants français ${ }^{58}$. Ce «comité de secours aux sinistrés d’Orléansville» intervient à Niamey et à Maradi sous les patronages respectifs du gouverneur et du commandant de cercle ${ }^{59}$. Il y est suggéré de « faire marcher le micro haut-parleur du cercle, d'employer le crieur public et le tam-tam des chefs » pour récolter des fonds, de suivre les comités de soutien en brousse où les personnalités religieuses musulmanes et chrétiennes sont enjointes de solliciter la générosité des fidèles. Un appel «à toute la population nigérienne » pour soutenir les victimes par des dons «en nature ou en espèces» est publié par l’administration et affiché dans les lieux publics. Une somme de 52325 francs CFA est ainsi collectée à Filingué et présentée comme une « contribution spontanée des habitants de la subdivision $\aleph^{60}$. Paradoxalement, cette année 1954 est celle de «la farine de manioc», désignation djerma de la crise alimentaire qui affecte l'ouest du pays jusqu'en195561.

Il existe donc au Niger de l'après-guerre une aide privée récoltée au bénéfice de populations métropolitaines, aide sollicitée non seulement auprès des Français mais aussi de Nigériens, essentiellement les élites, plus marginalement les milieux populaires. Même si, de fait, cette aide repose d'abord sur les expatriés, elle inclut virtuellement dans le cercle de ses donateurs potentiels la population nigérienne.

\footnotetext{
54 AMR, Lettre du gouverneur du Niger au maire de Rosières (21/05/1948). L'invocation de l'abolition de l'esclavage n'est pas fortuite, puisqu'il s'agit d'une des grandes justifications de la colonisation, soit l'un des principaux motifs pour lesquels la population colonisée doit de la reconnaissance aux colonisateurs.

55 CAOM, 1affpol/347, Gouvernement général de l'AOF, Direction générale de l'instruction publique, Renseignements fournis pour l'Assemblée générale des Nations unies (1949).

56 AMR, Lettre des élèves de Birni N'Konni aux élèves de Rosières (29/04/1948).

${ }^{57}$ ANM, 2H1479, Lettre du gouverneur général aux gouverneurs de l'AOF (25/05/1940).

58 Orléansville est une ville créée au XIX siècle à partir d'un camp militaire français. Le séisme de 1954 provoque près de 1500 morts.

${ }^{59}$ Le gouverneur du Niger est alors encore Jean-François Toby, nommé l’année précédente à ce poste pour la quatrième et dernière fois.

${ }^{60}$ ANN, 2H1.7, Comité de soutien aux sinistrés d'Algérie, Procès-verbal de réunion (18/09/1954).

${ }^{61}$ B.A. GADO (1993 : 46-47).
} 


\section{La mobilisation d'un langage caritatif entre France et Afrique}

Cette aide privée mobilise un langage de type caritatif, avec son lexique et ses rituels propres. L'usage de pareil langage n'est pas nouveau dans les années $1940^{62}$. Mais il reste globalement réservé à un rapport de relative proximité entre donateurs et donataires. Il est alors assez rare de le voir mobilisé entre deux continents, sinon dans le cadre d'un rapport d'appartenance à une même communauté nationale ${ }^{63}$.

Ce langage a d'abord largement recours à l'image pour susciter la compassion des donateurs potentiels. De 1943 à 1945, le parrainage de Rosières, que la colonie du Niger ne peut plus assurer, est repris par la commune d'Yssingeaux. Celle-ci met en place, aux côtés des subventions publiques votées par le budget municipal, un système de collectes alimentées par les dons privés. À cette fin, la municipalité d'Yssingeaux demande à celle de Rosières de lui faire parvenir des photographies de la ville détruite, stipulant que « la vision des ruines de [la] localité ne pourra qu'inciter la sympathie et la charité des Yssingelais ${ }^{64} »$. À partir de 1945, le Niger retrouve son statut de marraine et, comme à Yssingeaux, le comité de soutien à Rosières a recours à l'image pour favoriser la solidarité des donateurs potentiels. Ce recours est jugé d'autant plus important que ces donateurs sont loin des donataires. Des photographies prises en mai et juin 1940, au moment de la bataille de la Somme, sont ainsi envoyées au Niger pour susciter la solidarité de ceux dont le territoire a été épargné par la guerre65. Elles sont exposées à Niamey au regard des participants français et nigériens des manifestations organisées au bénéfice de la commune détruite ${ }^{66}$.

La présentation sélective des victimes est un autre élément important de ce langage caritatif. Certaines catégories de bénéficiaires de l'aide sont mises en exergue davantage que d'autres. De manière générale, les dons collectés par le comité de soutien à Rosières sont destinés aux « indigents » ou aux «nécessiteux » de la commune, à charge des autorités municipales de désigner les indigents ou les nécessiteux en question. Mais il arrive que ces dons soient adressés prioritairement à des catégories caractérisées par la vulnérabilité et, en cela, particulièrement visées par la démarche caritative. Le comité de soutien à Rosières, lorsqu'il précise les destinataires privilégiés de ses dons, désigne ainsi « les enfants » et les « vieillards $»^{67}$. Cette précision s'adresse autant aux donateurs potentiels qu’à la municipalité de Rosières, l'évocation des victimes les plus fragilisées par la guerre donnant plus d'impact à l'appel à la générosité. Il arrive que ces bénéficiaires prioritaires soient désignés comme tels indépendamment des dommages subis pendant la guerre. Dans plusieurs de ces lettres, le maire de Rosières, André Masson, accuse ainsi une bonne réception de dons destinés aux personnes âgées de plus de 60 ans, « qu'elles soient sinistrées ou non ${ }^{68}$ », précisant que « tous les vieux appelés à bénéficier de la

\footnotetext{
${ }^{62}$ Ce langage s'inscrit dans une longue histoire de la charité, thème central dans les ouvrages consacrés à la prise en charge de la pauvreté en Europe, notamment de Michel Mollat pour le Moyen-âge et de Bronislaw Geremek pour l'époque moderne.

63 P. SASSIER (1990: 268-269).

64 AMR, Lettre du rédacteur du Mémorial, journal régional de St Etienne, au maire de Rosières (12/05/1943).

65 AMR, Lettre du maire de Rosières au gouverneur du Niger (19/02/1946).

66 AMR, Lettre du gouverneur du Niger au maire de Rosières (06/04/1946).

${ }^{67}$ AMR, Lettre du gouverneur du Niger au maire de Rosières (27/10/1948).

68 AMR, Lettre du gouverneur du Niger au maire de Rosières (14/01/1948).
} 
générosité des Nigériens seront très touchés ${ }^{69}$ » et rappelant qu'il s'agit de «la catégorie qui a le plus à souffrir, devant supporter l'augmentation du coût de la vie sans pouvoir travailler ${ }^{70}$ ». De même, à partir de 1947, une partie de ces dons transitent directement par l'école de Rosières afin de bénéficier à l'ensemble des écoliers, quelque degré qu'ils aient été affectés par la guerre ${ }^{71}$.

Ce langage caritatif utilise également un rituel dans les modalités de collectes de fonds. L'organisation de manifestations festives dont les bénéfices sont reversés aux nécessiteux fait partie de ces rituels. En 1948, à l'occasion du centenaire de l'abolition de l'esclavage, plusieurs écoles nigériennes de l'intérieur du pays organisent ainsi des kermesses ou des représentations théâtrales dont les recettes sont reversées à la commune picarde. C'est le cas de l'école primaire de Birni N’Konni qui transmet une lettre destinée «aux enfants de Rosières-en-Santerre » signée par les écoliers nigériens, lettre dans laquelle ils s'excusent de ce que « [leur] don est petit», assurant qu'ils « [auraient] voulu faire mieux $»^{72}$. Le choix de dates privilégiées pour effectuer les collectes ou verser les fonds est un autre de ces rituels. En 1948, la Croix Rouge de la colonie envoie des colis de café, de riz, de savon et de chocolat à Rosières « pour permettre aux enfants des écoles, aux vieillards et aux familles nécessiteuses de passer une agréable fête de Noël73 . La même année, une maison spécialisée de Dakar est mandatée par le comité de soutien à Rosières pour envoyer des chocolats destinés aux fêtes de fin d'année des indigents de la commune ${ }^{74}$. À partir de 1949, une partie des subventions annuelles envoyées par le Conseil général sert à acheter des jouets comme cadeaux de Noël aux écoliers de Rosières ${ }^{75}$. On retrouve là non seulement dans la date des dons mais aussi dans les produits donnés les éléments d'un rituel caritatif, classique en France métropolitaine mais plus inhabituel au Niger.

Ainsi, la campagne d'aide à Rosières organisée au Niger mobilise un langage caritatif convenu, un langage qui ressemble fortement à celui plus tard mis en œuvre dans le cadre des campagnes de solidarité allant du Nord au Sud mais que l'on peut voir, dans les années 1940, usité sur un territoire africain au bénéfice de populations métropolitaines.

\section{Les organisations caritatives an Niger}

Cet usage d'un discours caritatif à l'intention de populations métropolitaines nécessiteuses paraît paradoxal sur un territoire où la majeure partie des habitants parait elle-même vivre dans la nécessité. Cela s'inscrit cependant dans un contexte où la question de la pauvreté au Niger n'est que très peu prise en charge par des associations privées. Au sortir de la guerre, les organisations caritatives œuvrant au Niger pour des populations nigériennes restent marginales.

${ }^{69}$ AMR, Lettre du maire de Rosières au gouverneur du Niger, (19/11/1946).

${ }^{70}$ AMR, Lettre du maire de Rosières à l'évêque de Niamey, (17/09/1947).

71 AMR, Lettre du gouverneur du Niger au maire de Rosières (30/11/1947).

72 AMR, Lettre des élèves de Birni N'Konni aux élèves de Rosières (29/04/1948).

73 AMR, Lettre du gouverneur du Niger au maire de Rosières (27/10/1948).

${ }^{74}$ AMR, Lettre du gouverneur du Niger au maire de Rosières (06/12/1948).

75 AMR, Lettre du maire de Rosières au gouverneur du Niger (31/03/1949). 
Les organisations privées les plus anciennement installées en Afrique occidentale dans une optique d'assistance sont les missions catholiques ou protestantes. Leur activité sociale, mal connue de l'administration, reste limitée en $\mathrm{AOF}$ et plus encore au Niger que dans les colonies voisines ${ }^{76}$. Un rapport établi pour l'ONU sur les œuvres religieuses en AOF à la fin des années 1940 recense, en matière d'aide à l'enfance, 11 orphelinats et 45 infirmeries scolaires tenus par des catholiques et aucun établissement tenu par les protestants pour lesquels les statistiques ne sont pas à jour. Ce rapport conclut à une « faible activité dans le domaine social», précisant que «c'est par son influence religieuse et morale que l'Église chrétienne contribue sans doute le mieux $[\ldots]$ à la protection de l'enfance $\aleph^{77}$. Un autre rapport similaire recense, en matière de protection de la santé, 23 dispensaires privés, soit moins du dixième des dispensaires relevant du service de santé colonial ${ }^{78}$. Globalement, la prise en charge de la santé par les œuvres privées reste limitée en AOF et s'y concentre essentiellement en Côte d'Ivoire, au Soudan et au Dahomey ${ }^{79}$. Mis à part cette activité sanitaire, les œuvres privées interviennent dans le secteur éducatif, mais de manière particulièrement marginale au Niger. Ainsi, alors que le nombre d'enfants scolarisés dans des établissements primaires privés constitue environ le quart du nombre total d'écoliers en AOF, il n'en représente que le quarantième au Niger, colonie qui compte le plus bas taux de scolarisation de la Fédération ${ }^{80}$.

En dehors des missions, il existe des organisations privées non confessionnelles exerçant une activité de type caritatif au Niger. Il est difficile de les recenser, les premiers suivis centralisés des organisations non gouvernementales sur le territoire nigérien n'intervenant qu’à partir des années 197081. Mais leur nombre reste de toute façon très limité dans les années 1940. En 1935, une circulaire du ministère des colonies demande à tous les gouverneurs coloniaux de dresser la liste des œuvres sociales de protection de la maternité et de l'enfance, objets de prédilection de pareilles œuvres. Pour le Niger, seules deux organisations sont recensées, le Berceau africain et l'Association des Dames de France, toutes deux rattachées à la Croix Rouge ${ }^{82}$. Celle-ci, plus ancienne des organisations caritatives non confessionnelles, est présente en AOF sous forme de Croix Rouge française. C'est en 1946 qu'elle s'organise de manière autonome dans la Fédération en lien étroit avec la Direction générale du service de la santé. Elle s'investit alors essentiellement dans les visites médicales de nourrissons ${ }^{83}$. D'autres organisations privées s'installent ou se créent au Niger après la guerre, mais le phénomène reste limité jusqu’à la famine du début des années 1970. Une étude faite à l’issue de cette famine

76 J.R. DE BENOIST (1984).

${ }_{77} \mathrm{CAOM}, 1 \mathrm{affpol} / 346$, Gouvernement général de l'AOF, Rapport sur les œuvres catholiques et protestantes en matière de protection de l'enfance (1948).

${ }_{78} \mathrm{CAOM}, 1 \mathrm{affpol} / 347$, Gouvernement général de l'AOF, Direction générale de la santé publique, Renseignements fournis pour l'Assemblée générale des Nations unies (1948).

${ }^{79} \mathrm{CAOM}, 1 \mathrm{affpol} / 346$, Gouvernement général de l'AOF, Direction générale de la santé publique, Rapport sur la protection maternelle et infantile (1949).

${ }^{80}$ CAOM, 1affpol/347, Recteur de l'AOF, Rapport sur l'enseignement en AOF (1948).

81 A Niamey, un «groupement des agences privées» est ainsi mis en place dans les années 1970 pour coordonner le travail des principales organisations pendant la famine, avec en particulier Oxfam, Care, Caritas, Africare et Catholic Mission.

82 ANN, 2H1.7, Lettre du Service de santé au ministre des colonies (04/04/1935).

${ }^{83} \mathrm{CAOM}, 1 \mathrm{affpol} / 346$, Gouvernement général de l'AOF, Direction générale de la santé publique, Rapport sur la protection maternelle et infantile (1949). 
sur « une proportion significative des organisations engagées dans le développement ou l'assistance au Sahel » montre que la majorité de ces organisations sont de création ou d'implantation récente, moins d'une dizaine remontant en deçà des années $1970^{84}$.

Un troisième type d'organisation caritative est constitué des comités d'assistance aux victimes de guerre, sur le même modèle que le comité de soutien à Rosières. L'activité de ces organisations traduit l'ambivalence d'une activité caritative qui, menée sur le territoire africain, s'adresse d'abord à des populations métropolitaines avant de s'ouvrir aux populations locales. Juste après la guerre, plusieurs organisations se mettent en place au Niger pour participer aux secours aux sinistrés. En commémoration du 18 juin, des collectes annuelles sont ainsi organisées à l'intention des « CEuvres de la France libre ${ }^{85}$ ». En 1946, un comité local est constitué sous le patronage de la femme du général de Gaulle, avec pour mission d'organiser des souscriptions à l'intention des enfants indigents, essentiellement des orphelins de guerre ${ }^{86}$. La même année, le gouverneur général Pierre Cournarie promeut l'institution d'une «semaine de l'Alsace-Lorraine » dans la Fédération pour collecter des fonds aux bénéfices d'une région particulièrement impliquée dans le conflit ${ }^{87}$. Cependant, plus on s'éloigne de la guerre et moins ces organisations sont portées à réserver leur fonds à des victimes métropolitaines : elles y adjoignent alors des populations locales non touchées par la guerre mais dont le profil se rapproche de celui des premiers bénéficiaires métropolitains. L’Union des aveugles et des grands infirmes de guerre recueille ainsi chaque année des fonds sur le territoire nigérien après la guerre. D’après les termes de son président, il s'agit d'abord d'améliorer « les conditions d'existence misérable des aveugles et des grands infirmes » en France ${ }^{88}$. À partir de 1955, les collectes sont annualisées dans le cadre d'une journée des aveugles de France et de l'Union française. Mais la moitié des fonds récoltés revient désormais aux aveugles nigériens tandis que l'autre reste réservée aux aveugles de guerre ${ }^{89}$.

Le parrainage de Rosières par la colonie du Niger s'inscrit donc dans un cadre caritatif nigérien caractérisé par un faible nombre d'organisations et une activité qui, loin d'être réservée à la seule population nigérienne, vise également la population métropolitaine. Ce phénomène tend à diminuer au cours des années 1950, les organisations caritatives se consacrant de plus en plus prioritairement aux conditions de vie des populations locales.

En 1957, le maire de Rosières-en-Santerre envoie ses vœux de bonheur et de prospérité à l'intention des « généreuses populations du Niger dont la ville de Rosières, désormais reconstruite et modernisée, n’oublie pas

84 B. Brown, J. Tuthill et T. Rowing (1976). Cette étude porte sur 63 organisations américaines, européennes, australiennes et africaines engagées dans les pays sahéliens. Sur les quarante organisations présentes au Niger - dont la moitié est fondée après les années 1940 -, dix arrivent dans les années 1950 ou 1960 et vingt dans les années 1970, la date d'arrivée des dix dernières n’étant pas précisée.

85 ANN, 2H1.7, Lettre du gouverneur du Niger au gouverneur général de l'AOF (22/07/1946).

86 ANN, 2H1.7, Circulaire du gouverneur du Niger aux commandants de cercle (12/01/1945).

${ }^{87}$ ANN, 2H1.7, Télégramme du gouverneur général aux gouverneurs de l'AOF (14/03/1946).

88 ANN, 2H1.7, Lettre du président de l'Union des aveugles et des grands infirmes au gouverneur du Niger (24/09/1954).

89 ANN, 2H1.7, Lettre du gouverneur général de l'AOF au gouverneur du Niger (10/12/1955). 
la grande générosité $»^{90}$. Seize années plus tard, au moment de la grande famine qui touche les pays sahéliens, le conseil municipal vote un crédit de 10000 francs et lance un appel à la charité publique pour porter secours aux populations du Niger ${ }^{91}$. Comme l'indique le principal quotidien nigérien, « les temps ont changé, Rosièresen-Santerre est une localité prospère $[\ldots]$ prête à venir en aide à des populations qui l'ont autrefois secourue $»^{92}$. Dans les années soixante-dix, le sens du rapport caritatif est devenu évident : entre un pays du Nord et un pays du Sud, il ne peut y avoir aucune ambiguiité sur la distribution des rôles d'aidant et d'aidé. Un pays comme le Niger se trouve structurellement défini par la pauvreté et son statut de pays aidé. À contrario, les pays industrialisés, et en particulier l'ancienne puissance coloniale, se trouvent investis du statut de pourvoyeurs de fonds, notamment dans les représentations collectives véhiculées au sein des pays aidés. À travers le parrainage de Rosières-en-Santerre par la colonie du Niger, on voit que cette évidence est le produit d'une construction encore mal établie dans les années 1940. Ce parrainage concourt à inscrire l'aide internationale, avec ses codes et ses représentations, dans une histoire. Le sens de cette aide n'est pas d'emblée aussi fléché et aussi unilatéral qu'il le devient par la suite.

\section{BIBLIOGRAPHIE}

Ageron Charles-Robert, Coquery-Vidrovitch Catherine, Meynier Gilbert, ThobIE Jacques, Histoire de la France coloniale, 1914-90, Paris, Armand Colin, 1990.

Ageron Charles-Robert, La décolonisation française, Paris, Armand Colin, 1991.

AKPO-VACHE Catherine, L'AOF et la Seconde guerre mondiale, Paris, Karthala, 1996.

BADO Jean-Paul, Médecine coloniale et grandes endémies en Afrique de l'Ouest, Karthala, 1996.

BRown Barbara, TUthill Janet, Rowe Thomas, International Disaster Response: the Sabelian Experience, University of Denver, Graduate School of International Studies, 1976.

CANTIER Jacques, Jennings Eric (éds.), L’Empire colonial sous Vichy, Paris, Odile Jacob, 2004.

Choquet Catherine, Dolfus Olivier, Le Roy Etienne, Vernieres Michel (éds.), État des savoirs sur le développement. Trois décennies de sciences sociales en langue française, Paris, Karthala, 1993.

CointeT-LaBrousse Michèle, Vichy et le fascisme, Bruxelles, Éditions Complexe, 1987.

ConkLIN Alice, A Mission to Civilize. The Republican Idea of Empire in France and West Africa, 1895-1930, Stanfort University Press, 1997.

90 ANN, 2H1.7, Lettre du maire de Rosières au gouverneur du Niger (12/02/1957). Le maire de Rosières est Raoul Régnier depuis mai 1953.

${ }_{91}$ AMR, Lettre du maire de Rosières au ministre de la défense nationale du Niger (18/05/1973).

92 ANN, Le Niger, 26/03/1973. 
COQUERY-VIDROVITCH Catherine, «Le financement de la « mise en valeur » coloniale : méthode et premiers résultats », Études africaines offertes à Henri Brunschwig, Paris, Éditions de l’EHESS, 1982.

COQUERY-VIDROVITCH Catherine, L'Afrique occidentale au temps des francais. Colonisateurs et colonisés, 1860-1960, Paris, La Découverte, 1992.

DE BENOIST Joseph Roger, Les relations entre l'administration coloniale et les missions catholiques au Soudan français et en Haute-Volta, thèse pour le doctorat d'État, Université de Paris 7, 1984.

DuluCQ Sophie, La France et les villes d'Afrique francophone. Quarante ans d'intervention (1945-1985), Paris, L'Harmattan, 1997.

ESCOBAR Arturo, Encountering Development. The Making and the Unmaking of the Third World, New-Jersey, Princetown University Press, 1995.

FIROUZEH Nahavandi (éd.), Repenser le développement et la coopération internationale. État des savoirs universitaires, Paris, Karthala, 2003.

GADO Boureima Alpha, Une histoire des famines an Sabel. Étude des grandes crises alimentaires (XIXe-XXe siècles), Paris, L’Harmattan, 1993.

GEREMEK Bronislaw, La potence et la pitié, Paris, Gallimard, 1987.

ILIFFE John, The African poor. A History, Cambridge University Press, 1987.

LABORIE Pierre, L'opinion française sous Vichy. Les Français et la crise d'identité nationale, 1936-1944, Paris, Le Seuil, 2001.

MERRIEN François-Xavier, L'Occident et les pauvres, bier et aujourd'bui, Paris, Les éditions de l'atelier, 1994.

Michel Marc, Les Africains et la Grande guerre. L'Appel à l'Afrique (1914-1918), Paris, Karthala, 2003.

MolLAt Michel, Les pauvres au Moyen-âge : étude sociale, Paris, Hachette, 1978.

RIST Gilbert, Le développement. Histoire d'une croyance occidentale, Paris, Presses de la fondation nationale de sciences politiques, 1996.

SASSIER Philippe, Du bon usage des pauvres. Histoire d'une théorie politique, XVIe-XIXe siècle, Paris, Fayard, 1990. 